\title{
Bricologie. La souris et le perroquet
}

Retour sur une exposition

\section{Thomas Golsenne}

\section{(2) OpenEdition \\ Journals}

Édition électronique

URL : https://journals.openedition.org/tc/7576

DOI : $10.4000 /$ tc. 7576

ISBN : 0248-6016

ISSN : 1952-420X

Éditeur

Éditions de l'EHESS

\section{Édition imprimée}

Date de publication : 24 décembre 2015

Pagination : 128-151

ISBN : 978-2-7132-2505-5

ISSN : 0248-6016

Référence électronique

Thomas Golsenne, « Bricologie. La souris et le perroquet », Techniques \& Culture [En ligne], 64 | 2015, mis en ligne le 24 décembre 2018, consulté le 29 septembre 2022. URL : http://

journals.openedition.org/tc/7576; DOI : https://doi.org/10.4000/tc.7576 


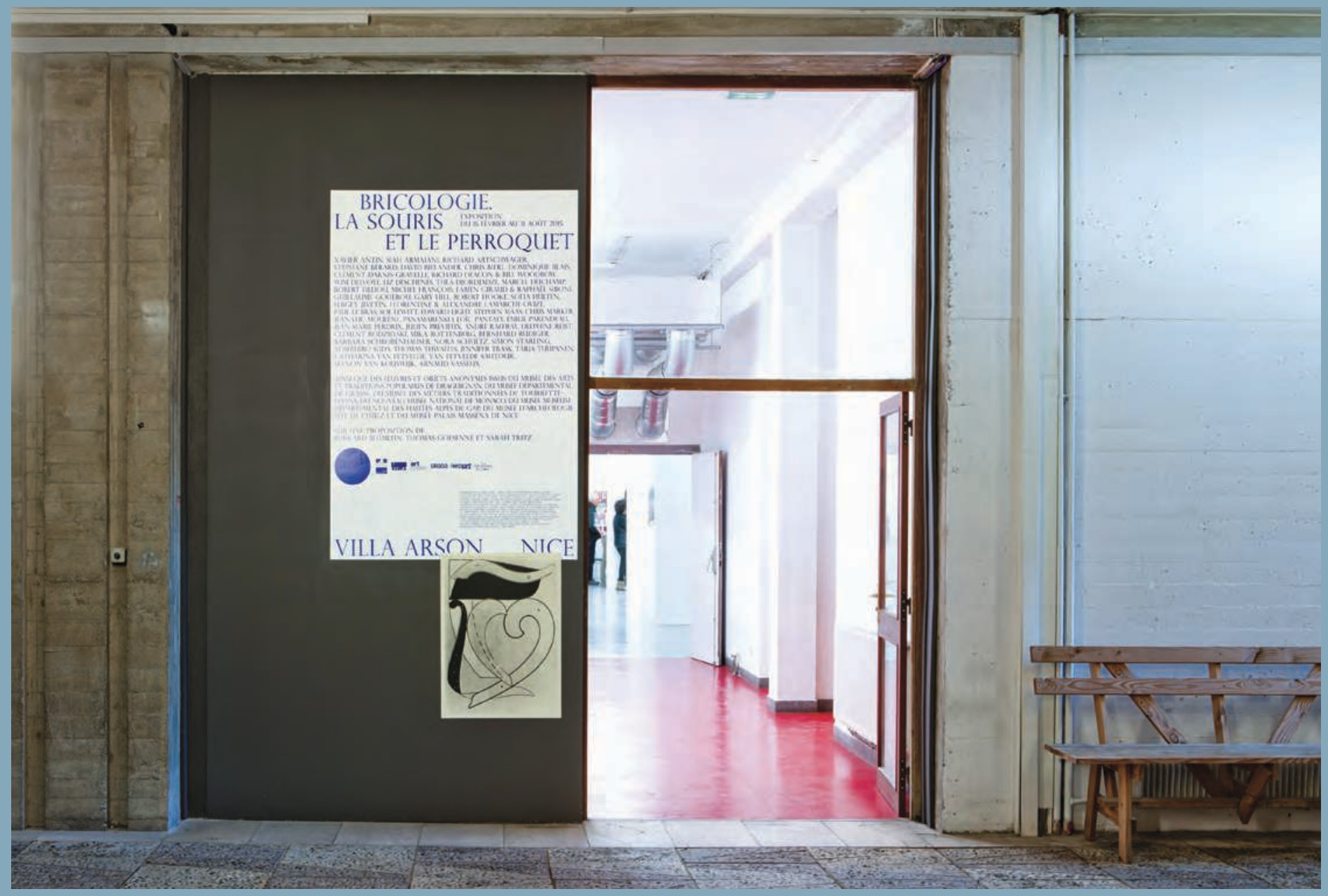




\section{Bricologie. La souris et le perroquet}

\section{Retour sur une exposition}

L'exposition Bricologie. La souris et le perroquet (Centre national d'art contemporain de la Villa Arson, Nice, 14 février - 31 août 2015) fut le résultat de deux années de recherches au sein de la Villa Arson (École nationale supérieure d'art de Nice) animée par trois enseignants dont deux artistes, Burkard Blümlein, Sarah Tritz et moi-même, historien de l'art, et suivie par une trentaine d'étudiants. Elle s'inscrivait dans l'Unité de recherche « Bricologie » qui engage d'autres projets, dont ce numéro de Techniques\&Culture.

La Souris et le perroquet était une exposition collective, regroupant une quarantaine d'artistes et une vingtaine d'objets d'artisanat puisés dans les riches collections des musées de la région niçoise. Contrairement à la plupart des expositions collectives qui portent soit sur un mouvement, soit sur un thème extérieur ou préalable aux cuvres dont la signification est comprise comme une réaction à ce thème, nous avons voulu montrer que la fabrication même des œuvres d'art est porteuse de sens. Tandis qu'habituellement on cache les questions techniques comme un parent gênant dont on a honte pendant une fête, comme des outils dans un placard une fois l'accrochage terminé, nous avons souhaité mettre en valeur les choix techniques opérés par les artistes, comme autant de façons de tracer un chemin singulier dans la création. Lartiste fabrique-t-il lui-même ses pièces ou en laisse-t-il la réalisation à d'autres, plus compétents que lui dans la maîtrise du savoir-faire requis? Achète-t-il ses outils dans le commerce ou les fabrique-t-il lui-même? Est-il à l'affût des nouvelles technologiques ou se complaît-il à exhumer des techniques oubliées? Se définit-il comme un bricoleur ou un ingénieur? Toutes ces questions, nous les avons posées à beaucoup d'artistes vivants, dont un grand nombre montrent leurs pièces dans l'exposition.

Mais à la rigueur n'importe quel artiste pourrait donner sa réponse à ces interrogations. C'est dire, en d'autres termes, le paradoxe de la technique : elle est partout bien qu'elle reste dans l'ombre de l'art. Aussi, pour restreindre notre sélection, nous avons choisi des artistes et des œuvres qui 
ne se contentaient pas d'utiliser des techniques particulières, mais qui faisaient de la technique l'enjeu principal de leur travail. La technique n'est plus seulement un moyen, mais une fin. Comprendre comment l'œuvre est faite, saisir sa « chaîne opératoire », pour parler comme André Leroi-Gourhan, est le seul moyen d'avoir accès à sa signification, qui peut porter, selon les cas, sur la question de l'artisanat, sur le bricolage, sur les gestes manuels, sur l'importance du processus, sur la matérialisation des projets, sur le rôle des outils, sur l'enchantement des technologies ou sur les limites de la virtuosité. Ces problématiques correspondaient aux zones à partir desquelles nous avons organisé l'exposition, en regroupant les artistes qui y répondaient à leur manière.

L'exposition se voulait aussi un hommage à Dédale, l'inventeur du labyrinthe et des automates, le premier ingénieur, patron des artisans en Grèce ancienne. Dans un livre remarquable, l'historienne Françoise Frontisi-Ducroux (2000 [1975]) dresse le portrait du héros des techniciens qui, dans une culture où l'artisanat était mal considéré et où l'art ne se différenciait pas de la technique, incarnait la valeur intellectuelle la plus prisée des Grecs : la ruse (Detienne \& Vernant 2013 [1934]). La ruse s'oppose à la force en tant qu'elle choisit la voie oblique, le détour, pour parvenir à ses fins. Une technique rusée n'est pas le moyen le plus direct de produire le résultat voulu, mais le plus surprenant, le plus inventif. Or Dédale utilise toujours la technique et son inventivité pour résoudre un problème, pour se sortir d'un mauvais pas, pour tromper ses ennemis - en rusant. Le labyrinthe est la figure architecturale de la technique rusée. L'histoire des techniques, de ce point de vue, n'est pas une succession linéaire d'inventions, mais un dédale proliférant de solutions inattendues face à des impasses, des erreurs, des besoins nouveaux. Et l'histoire de l'art également. S'il y a bien quelque chose de commun aux artistes modernes et à leurs successeurs contemporains, malgré l'immense diversité de leurs approches de l'art, c'est l'adoption du détournement comme méthode (de techniques, de matériaux, d'objets et d'images). Les artistes ont choisi la voie de Dédale et la ruse comme éthique. Nous avons essayé de présenter quelques-uns de ces héritiers de Dédale dans cette exposition. Nous avons voulu, de plus, les faire dialoguer avec d'autres héritiers, artisans ou bricoleurs anonymes, d'hier et d'aujourd'hui, dont les objets figurent dans les collections des musées des arts et traditions populaires. Ils partagent avec les artistes moins des formes et des problèmes que des procédures et ce sens de la ruse qui transforme le plus modeste objet technique en œuvre d'art. Parfois en effet, l'ingéniosité, dont font preuve les bricoleurs anonymes, témoigne moins d'une volonté de produire un outil efficace qu'un objet singulier. Dans d'autres cas, c'est le regard que les artistes portent sur des outils anciens, dont l'usage a été oublié, qui leur confère une valeur esthétique qu'ils n'avaient pas forcément au départ.

Le centre d'art de la Villa Arson se prêtait admirablement à un tel projet. Communément dénommée « le labyrinthe », la partie la plus étendue de ses espaces est une merveille de complexité, avec ses niveaux et ses escaliers, ses passages et ses recoins, ses enfilades et ses impasses. C'est tout naturellement que nous avons profité de cette configuration pour organiser le parcours du visiteur de telle sorte qu'il ne soit pas linéaire mais multiple, avec deux entrées et une succession non chronologique des pièces. J'ai reconstitué ici une visite guidée virtuelle et partielle de l'exposition, à travers toutes ses sections. 


\section{Artisanat}

Être qualifié d'artisan, pour un artiste, c'est souvent proche de l'insulte. Il ne faut lui en vouloir : depuis des lustres, la figure de l'artiste a été construite dans l'histoire de la pensée européenne contre celle de l'artisan. Depuis que la peinture est une « cosa mentale », comme disait Léonard de Vinci, les beaux-arts se sont spiritualisés, par distinction avec les arts et métiers. Mais dans
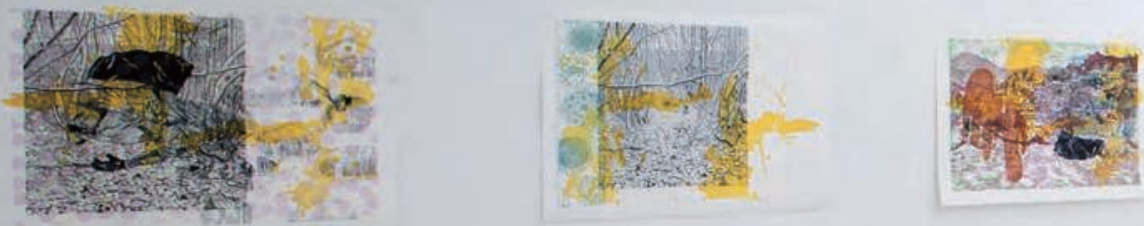

1. Alexandre Ovize \& Florentine Larmarche : Potacrayons, 2014 (faïence émaillée) et Lithographies, 2014 (eau-forte, lithographie et linogravure sur papier). LamarcheOvize sont des amateurs, dans les deux sens du terme : ils aiment explorer des techniques qu'ils apprennent en autodidactes, avec joie et décontraction. 
le monde contemporain, les cartes ont été rebattues, l'opposition n'est plus aussi franche. D'un côté, certains artistes ont retrouvé les joies du « fait main », les plaisirs d'une technique bien mâ̂trisée, comme la céramique qui a fait son grand retour dans l'art depuis une dizaine d'années; de l'autre, ont émergé les « métiers d'art » et les « maîtres d'art » qui aspirent aujourd'hui à un statut équivalent à celui des artistes, comme le montre le cas particulier des bijoutiers contemporains. On découvre ainsi le domaine de la «techno-esthétique », défriché par le philosophe Gilbert Simondon (2014 [1982]), celui où les outils sont beaux et pas simplement pratiques.

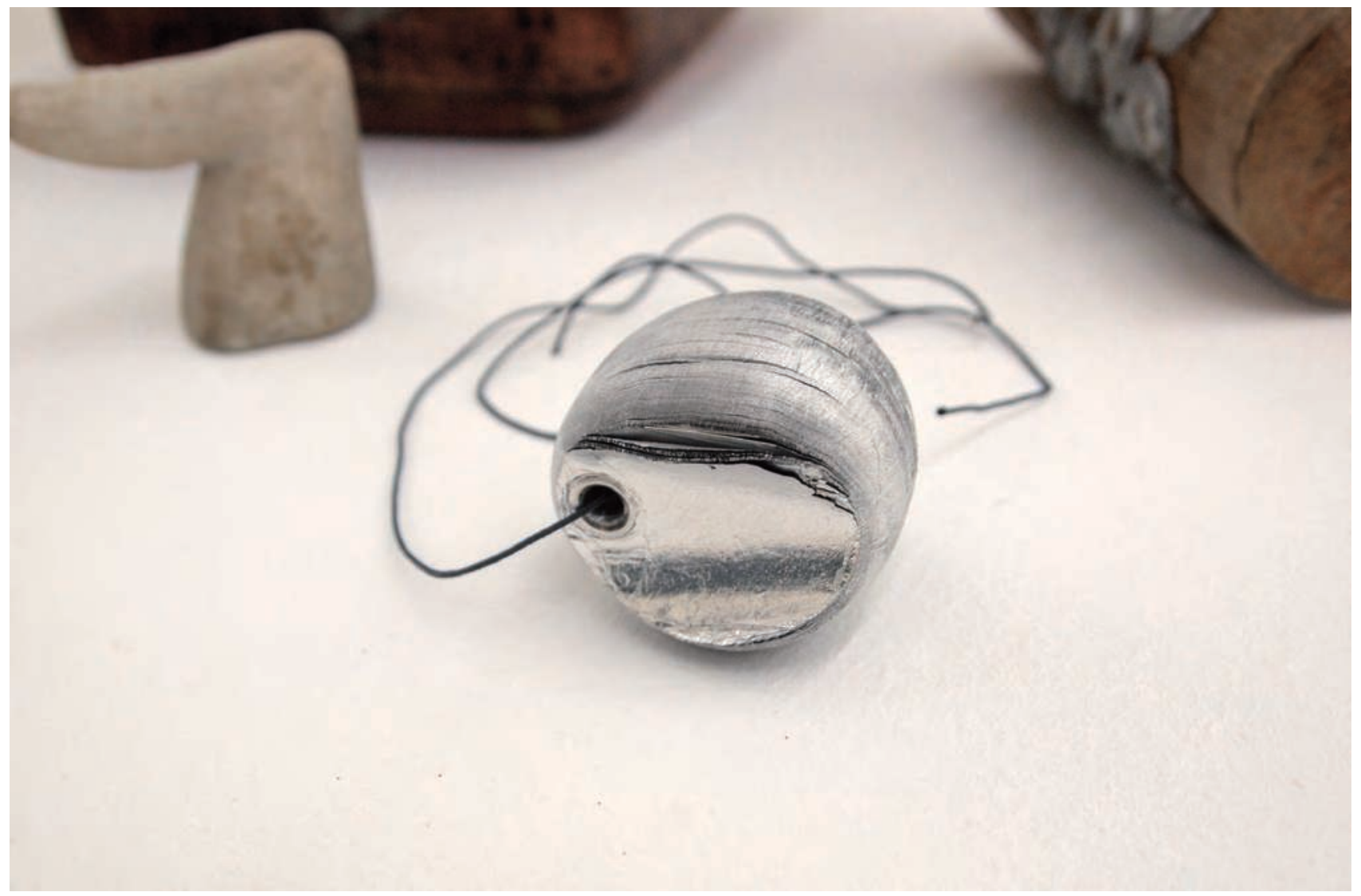

2. Barbara Schrobenhauser : Aluminium II (collier, aluminium, corde)

Schrobenhauser est représentative d'une génération de jeunes bijoutiers qui n'ont plus besoin des matériaux précieux de la joaillerie pour créer des bijoux très élaborés. 


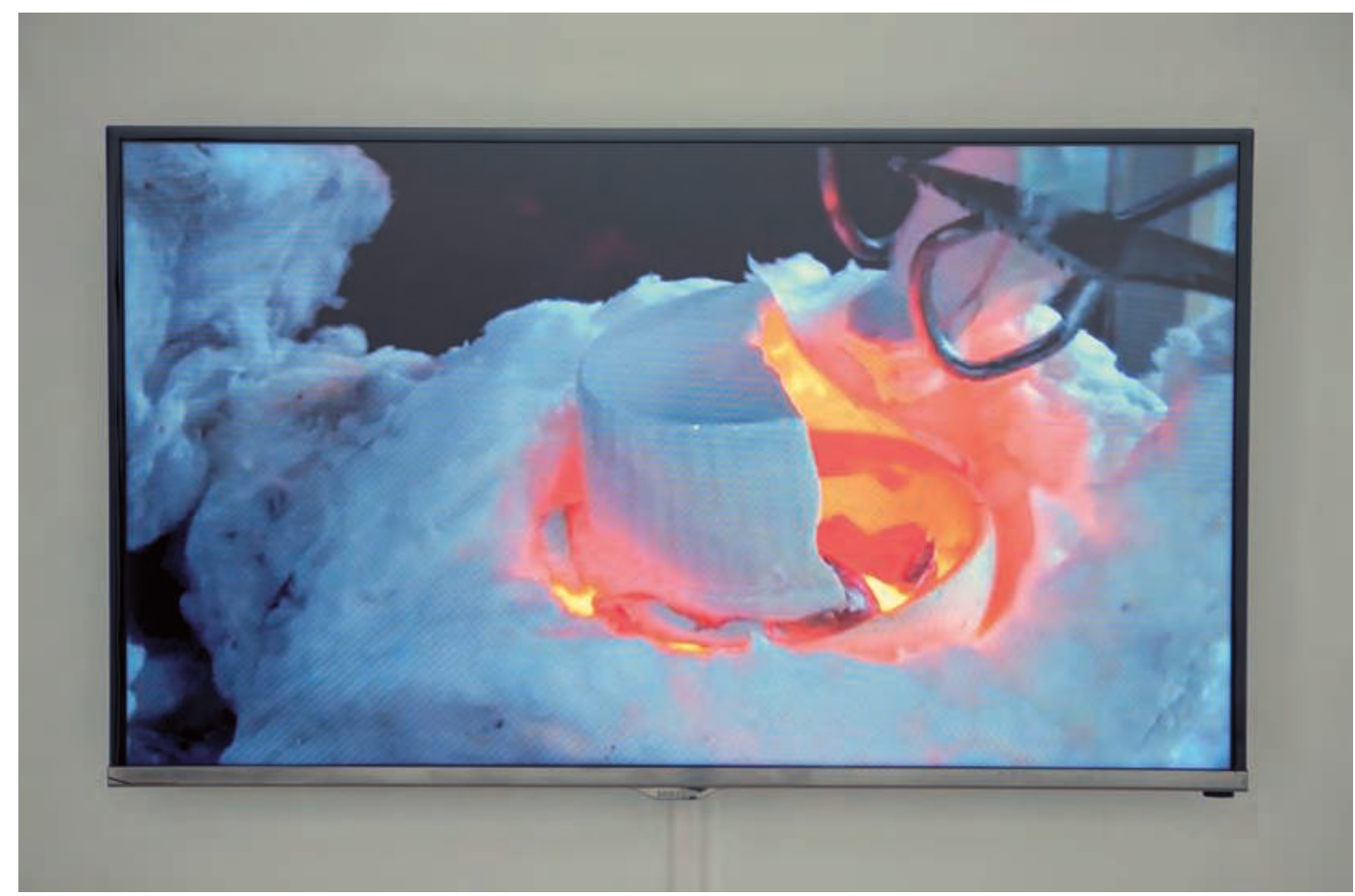

\section{Bricolage}

Le bricolage est une pratique noble, pour peu qu'on reprenne les idées affirmées par Claude Lévi-Strauss dans La Pensée sauvage (1962). Le grand anthropologue écrivait que le bricolage est une alternative au travail de l'ingénieur, une façon de penser des projets et des idées non dans l'abstrait, mais dans le concret, à partir d'un stock d'objets trouvés constitué au fur et à mesure. L'ingénieur, lui, construit les objets nécessaires à la réalisation de son idée. La différence : chez ce dernier, la réalisation correspond en tout point au projet, tandis que chez le bricoleur subsiste un écart entre les deux, du fait de la contingence de ses outils et de ses matériaux. Mais dans cet écart peut apparaître une heureuse surprise, une véritable invention. Dans ce sens, le bricolage constitue l'économie de travail de tous les amateurs, des partisans du do-it-yourself dont internet, les fabs labs ou les makers faires regorgent, mais aussi de bon nombre d'artistes contemporains qui jouent avec les objets, les matériaux et les techniques, en « amateurs » et avec liberté.
3. Thomas Thwaites: The Toaster Project, 2011 (vidéo documentaire). Le film de Thwaites relate les étapes d'un projet fou : refaire, tout seul, un grille-pain bon marché, quitte à devoir aller au fin fond de l'Écosse dans des mines de mica pour en extraire le minerai isolant. 


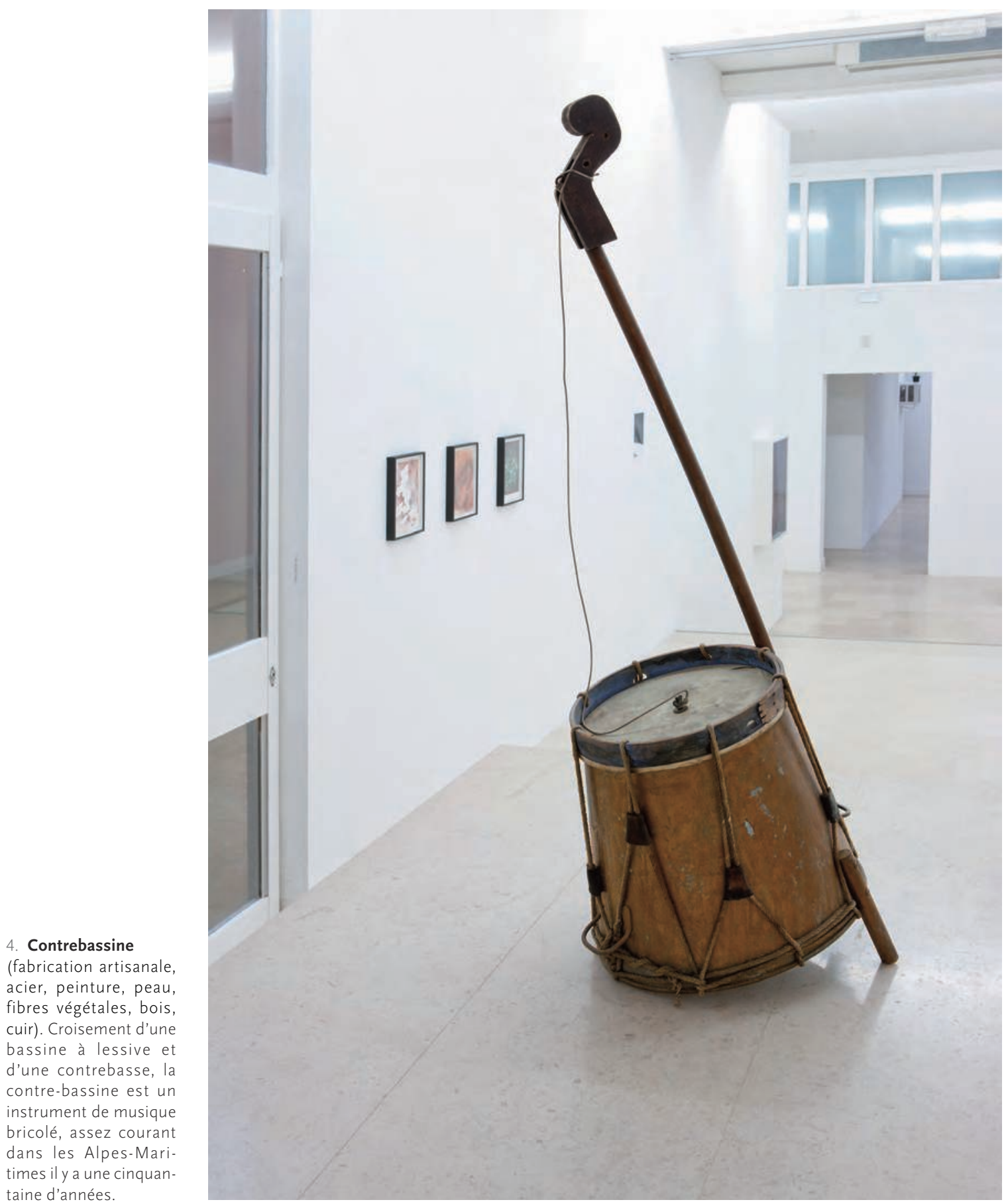




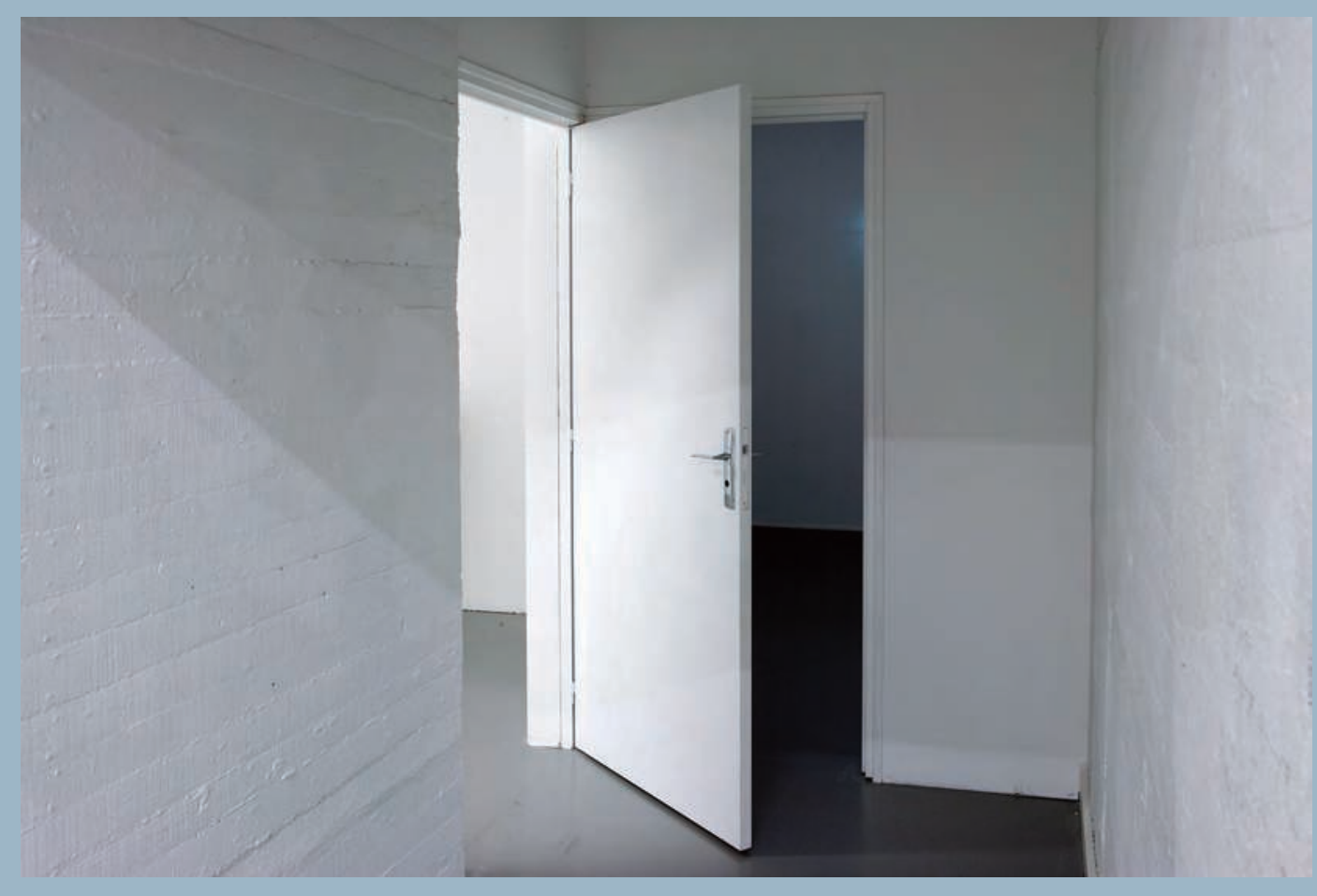

\begin{abstract}
5. Marcel Duchamp Porte, 11 rue Larrey, 1927 (ready made in situ, réactivé pour l'exposition). Son épouse d'alors le définissait non comme un artiste, mais comme un bricoleur. La porte de leur appartement rue Larrey, qui ouvre et ferme en même temps deux salles, est autant une trouvaille ingénieuse qu'une pièce conceptuelle.
\end{abstract}

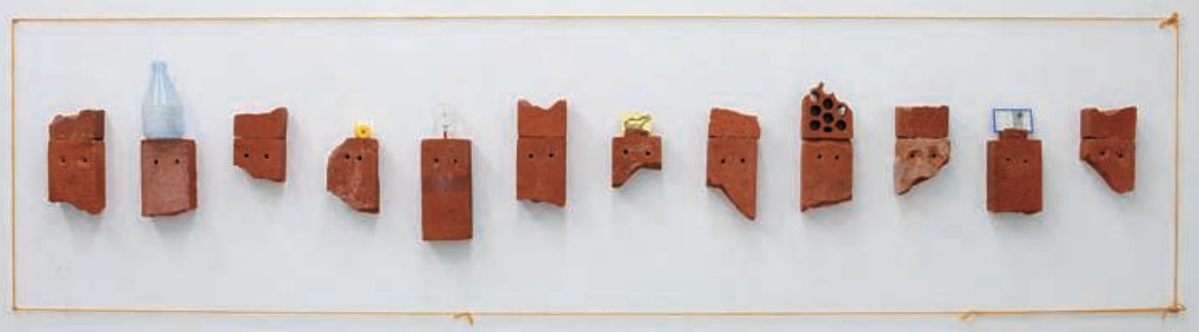

\begin{abstract}
6. Robert Filliou Briquolage I, 1982 (installation composée de 26 briques, 2 loupes, 2 miroirs, 5 dés, 2 bouteilles, 4 bouts de ficelle réunis, 1 rondelle). Le jeu de mots de cette pièce cache le sérieux d'une conception de l'art dans laquelle ce sont les objets trouvés qui donnent des idées, et non le contraire.
\end{abstract}


7. Guillaume Gouerou : Metatron Project MW6400, 2013-2014 (four à micro-ondes géant et tableau de commande). Ancien étudiant de la Villa Arson, Gouerou se sert du bricolage pour développer un univers de science-fiction et renouveler la figure de l'inventeur prêt à faire exploser son laboratoire pour aller jusqu'au bout de ses idées.

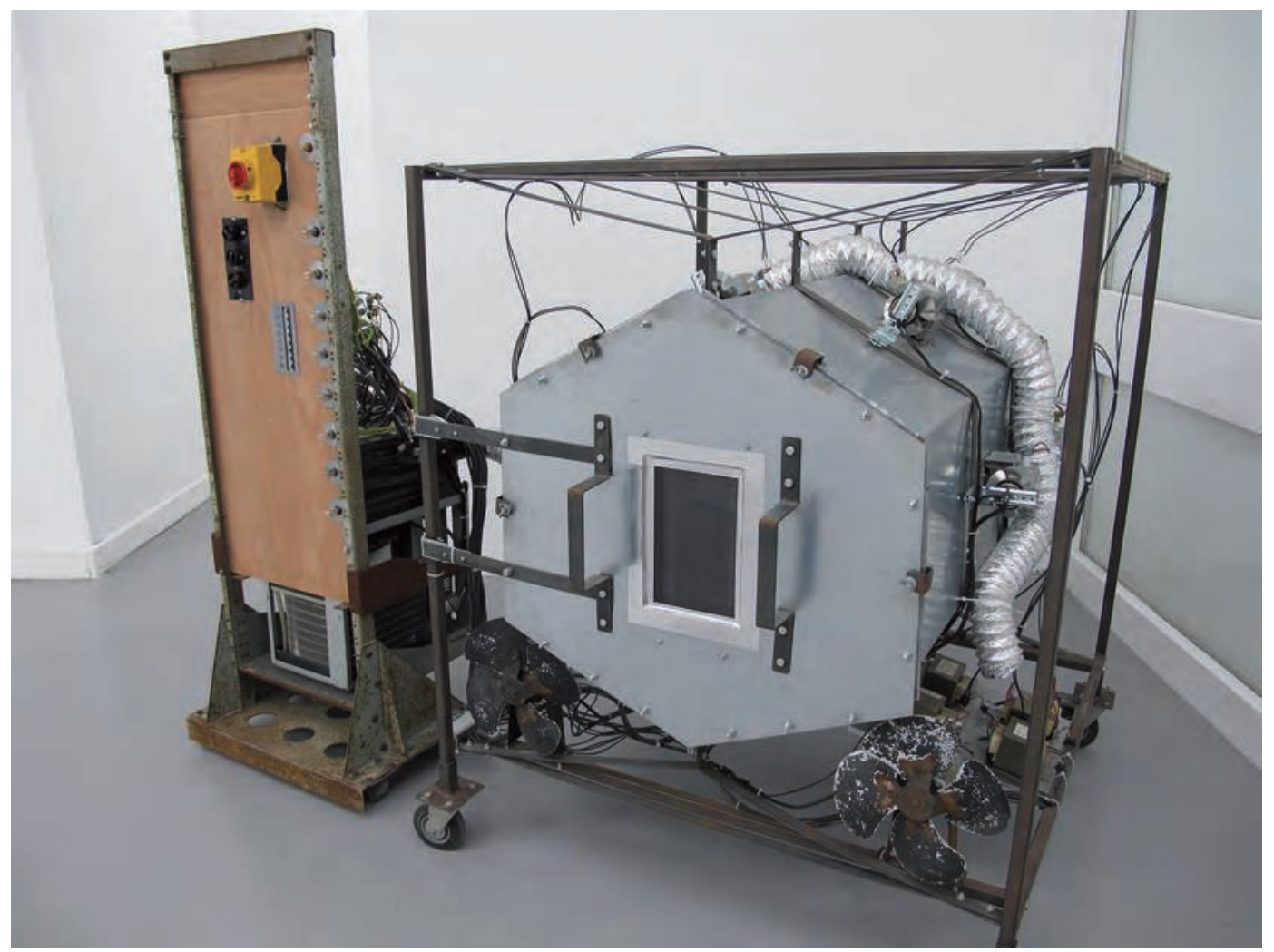

\section{Geste}

Leroi-Gourhan et les paléoanthropologues à sa suite estiment que le développement du cerveau est relié à celui de la main : l'humain moderne est apparu non pas seulement avec l'homo sapiens, mais avec l'homo faber, celui qui fabrique: les gestes de la main sont les premiers signes de l'intelligence. Aussi ne peut-on pas dire que la main est la simple servante de l'esprit : connaître le monde, c'est développer un «flair tactile», écrit magnifiquement Henri Focillon dans son Éloge de la main (1934). Et il continue : « L'art se fait avec les mains. Elles sont l'instrument de la création, mais d'abord l'organe de la connaissance. [...] C'est que [l'artiste] recommence toutes les expériences primitives : comme le Centaure, il tente les sources et les souffles. Tandis que nous recevons le contact avec passivité, il le recherche, il l'éprouve. Nous nous contentons d'un acquis millénaire, d'une connaissance automatique, et peut-être usée, enfouie en nous. Il la ramène à l'air libre, il la renouvelle - il part du début. » 


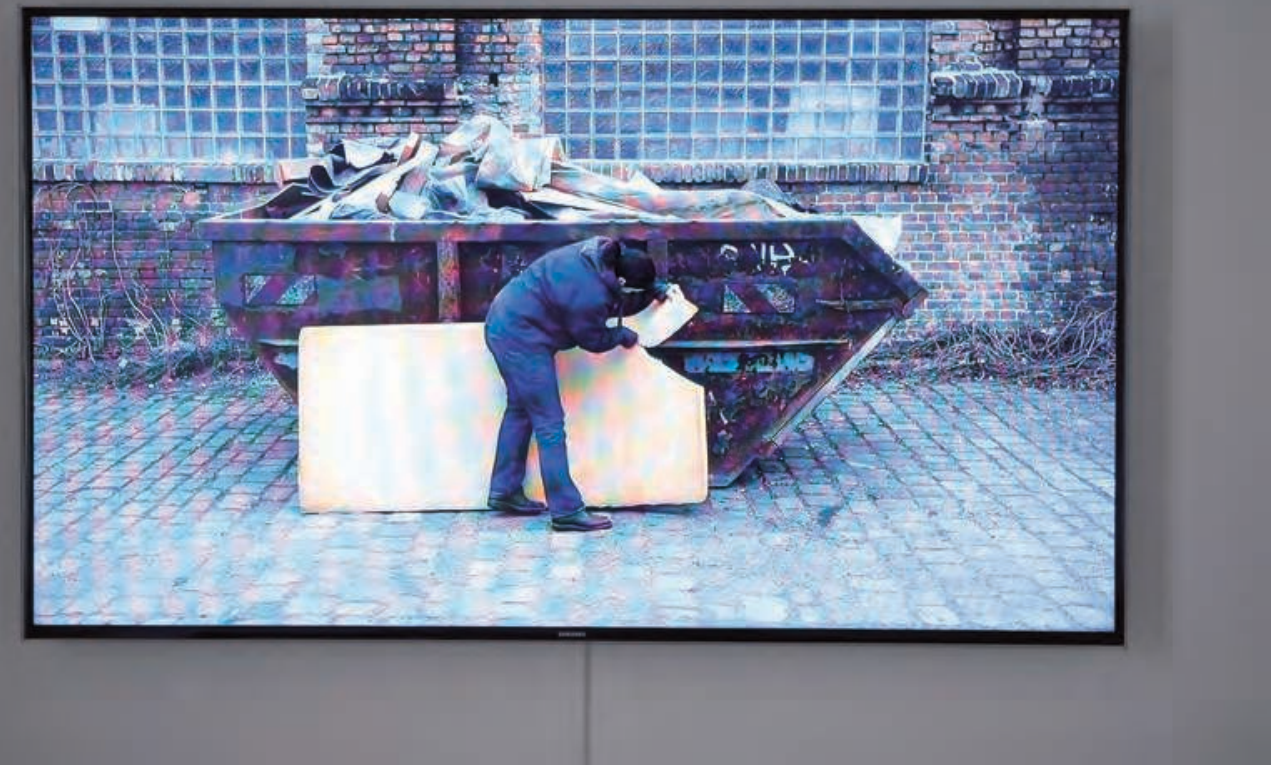

8. Sofia Hultén : Altered Fates, 2013 (vidéo sonore sur écran plat, 9 $\min 33 \mathrm{sec}$ ). La benne à ordures devient la métaphore de l'atelier de l'artiste, où celle-ci manipule des matériaux tirés au hasard, déroulant tout le vocabulaire des gestes du sculpteur.

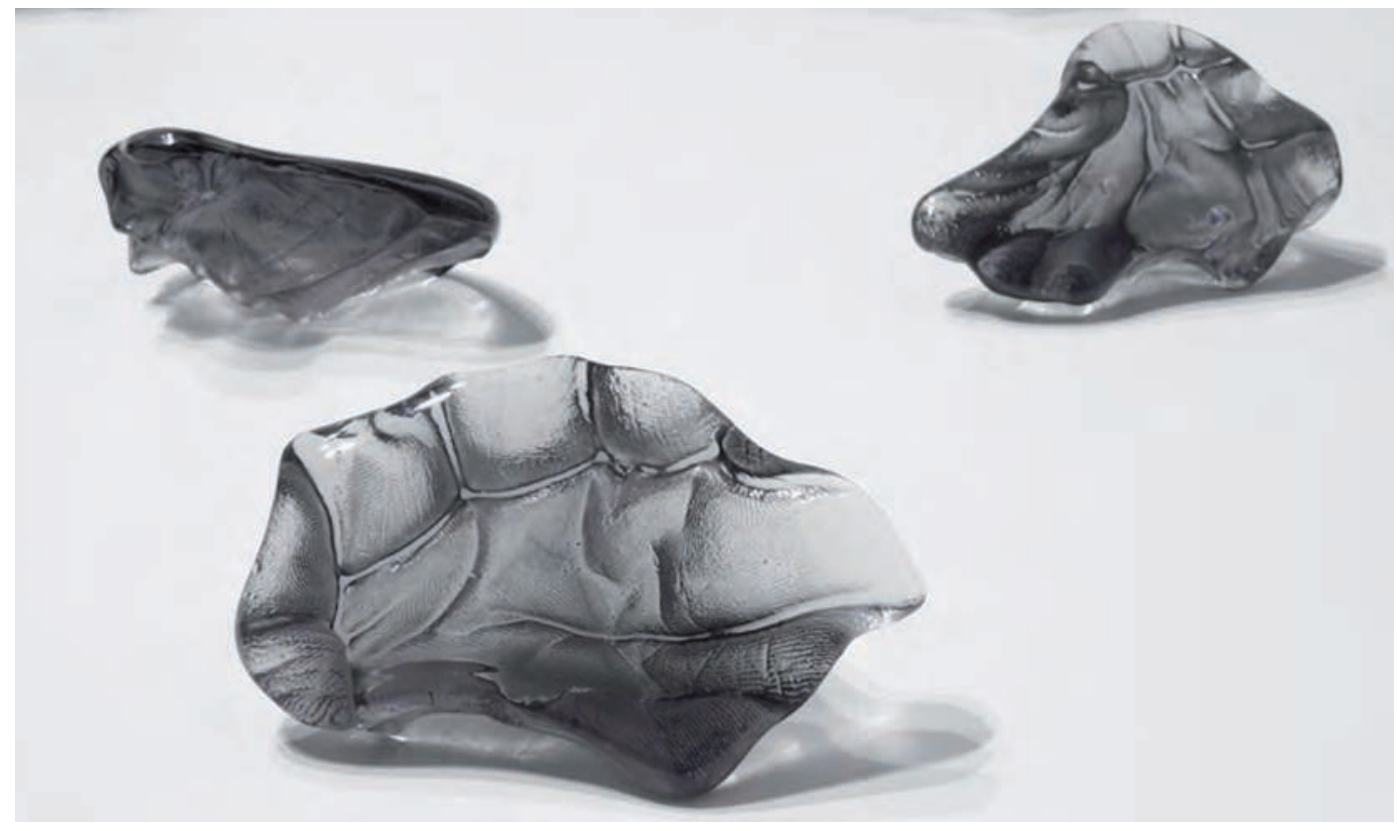

9. Arnaud Vasseux Creux, 2011-2013 (verre). L'artiste a moulé en résine la main des souffleurs de verre du CIRVA et en a tiré des formes de verre, rendant ainsi hommage au premier instrument de ces techniciens hors pair. 
10. Vitrine du geste (anonyme) : Statue de saint Marc de NotreDame de Brusc (bois peint) ; silex taillé (période du chalcolithique) ; massette (date inconnue). La statue de saint Marc, grattée par les pèlerins, la massette de métal, aux bords enfoncés par les coups et la « livre de beurre » qui a servi de matrice à lames, portent les traces de gestes élémentaires.

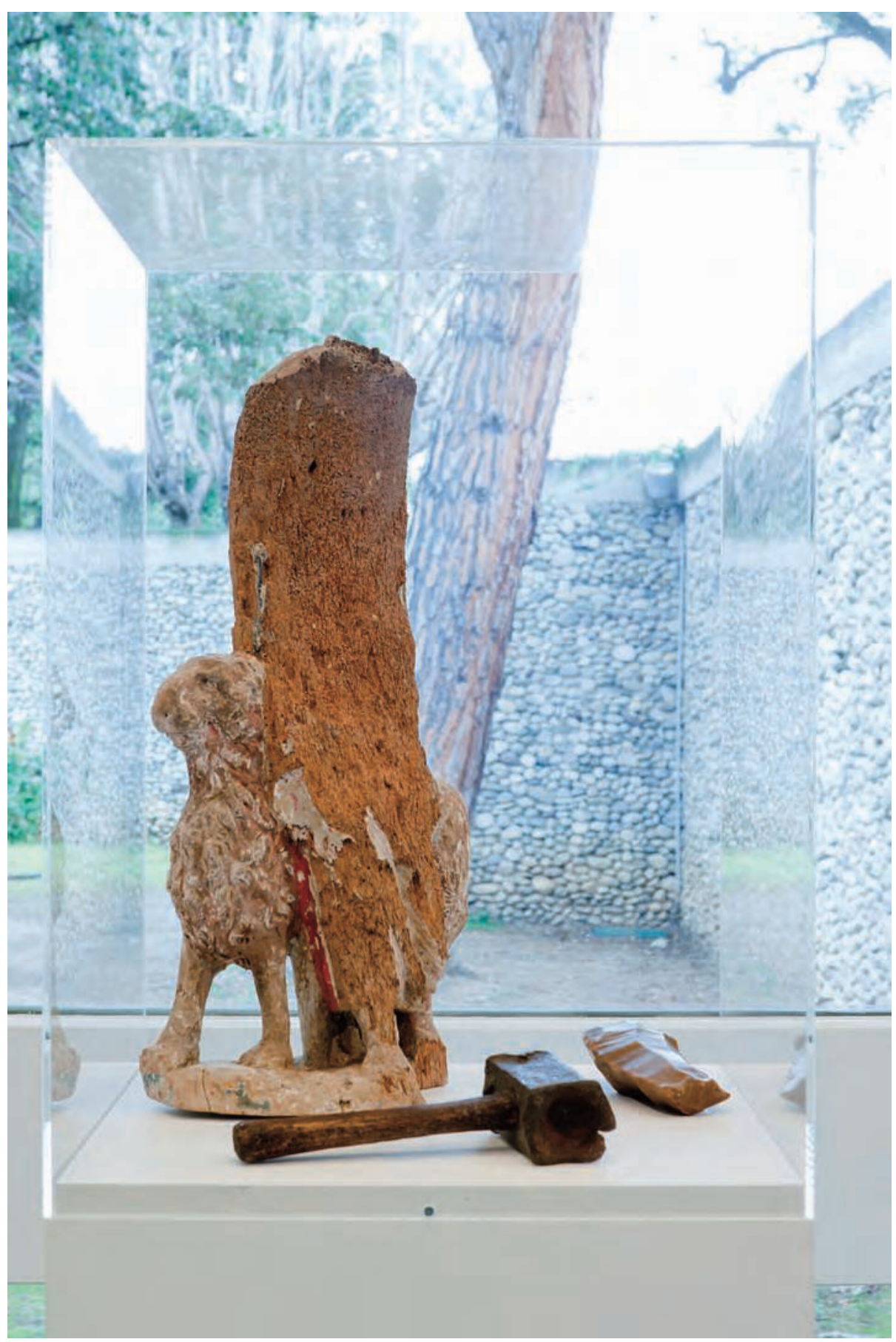




\section{Magie}

Qu'est-ce qu'un prestidigitateur? Littéralement, c'est celui qui a des doigts prestes. On l'appelle couramment magicien car il semble accomplir des merveilles. En fait, plus grande est sa maîtrise technique, plus elle se dissimule. À la Renaissance, l’art de cacher l'art était le nec plus ultra de la perfection dans l'accomplissement d'une pratique, qu'il s'agisse de la peinture, de la danse ou de la séduction. Art, technique et magie étaient alors étroitement liés; on pouvait faire l'expérience de la camera obscura où des images apparaissaient sans qu'on en devine l'origine, en appliquant de simples principes optiques, ou d'un trompe-l'œil de peinture, comme autant de merveilles où la nature et l'art se confondent. Certains artistes contemporains semblent perpétuer à leur manière cet esprit d'émerveillement et trouver dans le paradoxe d'une technique invisible l'origine de la fascination que leurs objets produisent (Gell 1992).

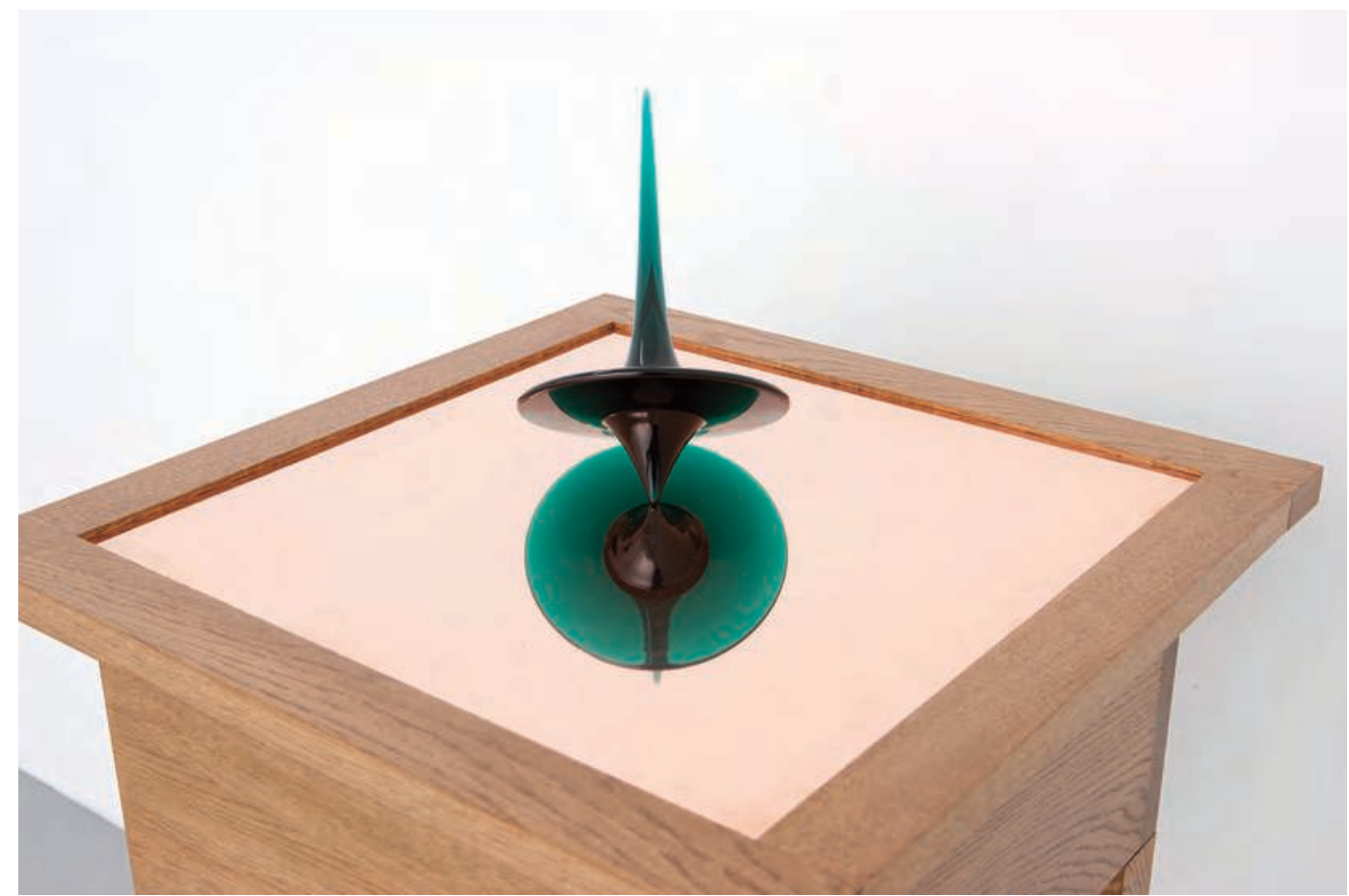

11. Dominique Blais : Entropê, 2014-2015 (verre, oxyde de cuivre, cuivre, chêne massif)

La toupie en verre de Dominique Blais, dont la forme lui a été inspirée par le mouvement rotatoire de la canne des souffleurs, évoque également les résistances des lignes à haute tension et la propriété curieuse de ce matériau, conducteur d'électricité seulement à chaud. 


\section{Processus}

Pour schématiser, on dira qu'il existe trois catégories d'artistes : ceux pour qui le résultat final compte avant tout, ceux pour qui le chemin, le processus qui y mène est plus important, et ceux qui accordent autant d'importance au processus qu'au résultat. Si les premiers artistes dominent l'histoire de l'art ancien, les seconds et les derniers prennent le dessus à partir de l'après 1945, mais la tendance commençait déjà à se renverser avec les avant-gardes depuis Cézanne. Il ne s'agit pas cependant que d'une question historique, mais bien d'une façon très différente de concevoir le but, le sens du travail artistique. L'œuvre processuelle est la trace d'une série d'actions, d'une chaîne opératoire définie par l'artiste, qui peut parfois prendre la forme d'une véritable performance. C'est sur cette chaîne opératoire que l'analyse doit se porter pour comprendre les artistes qui travaillent de cette manière. Parfois le processus continue alors même que l'œuvre est installée. Il faut donc voir, dans les pièces présentées dans cette section, non pas seulement un ensemble de formes, mais le passage du temps qui les transforme.

12. Xavier Antin : Sans titre (An Epoch of Rest), 2014 (tubes métal laqués, plastique, coton sergé, impression jet d'encre) et Sans titre (An Epoch of Rest), 2015 (tubes métal laqués, plastique, tabouret d'atelier, scanner A4, vidéo-projecteur, vidéo HD 25mn). Un projecteur diffuse des images de fleurs sur un scanner. lequel, relié à une imprimante arrangée par l'artiste, imprime de grands lavis ornementaux sur des tissus : tels sont les éléments de la chaîne opératoire que montre Xavier Antin.

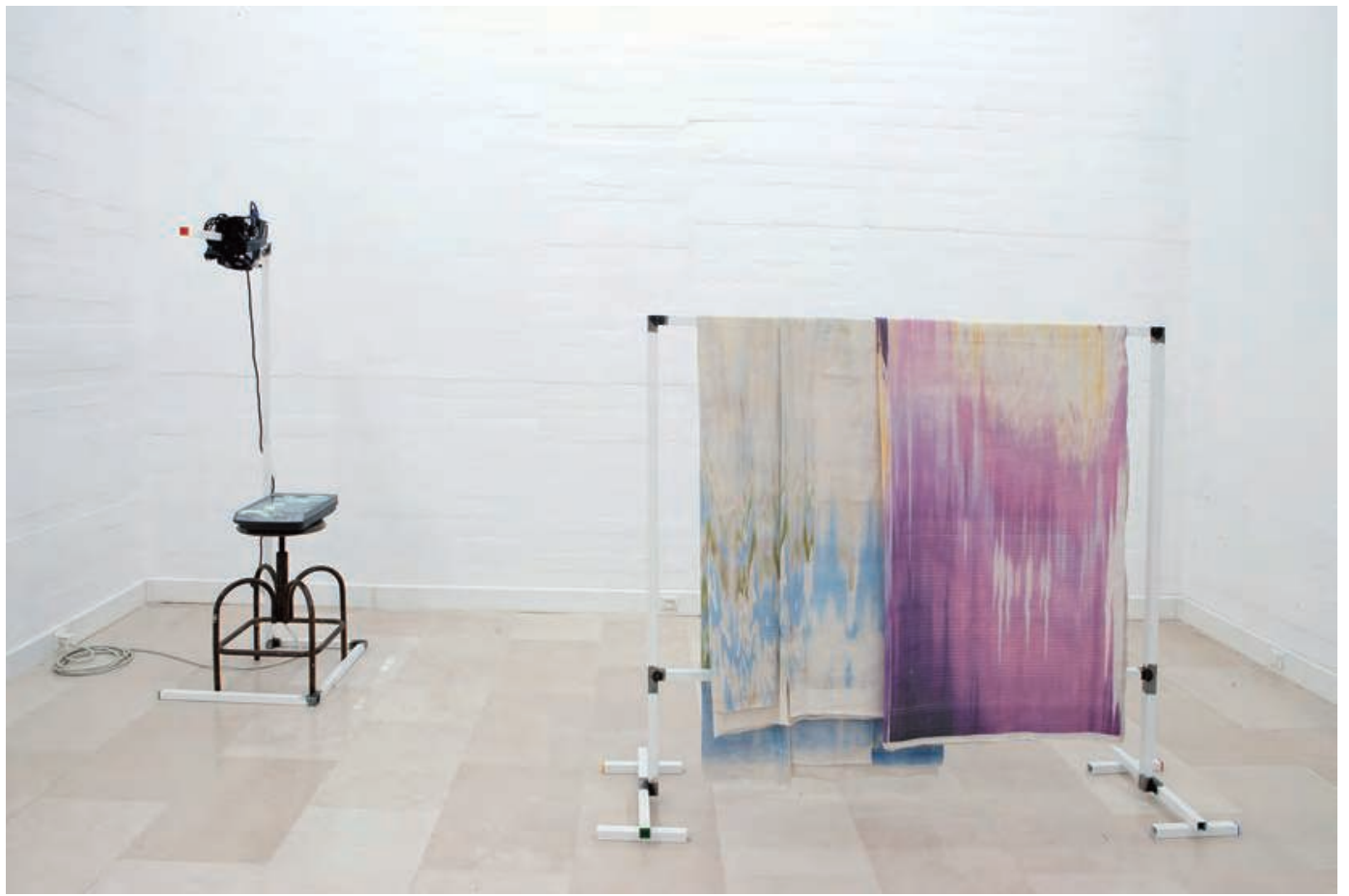




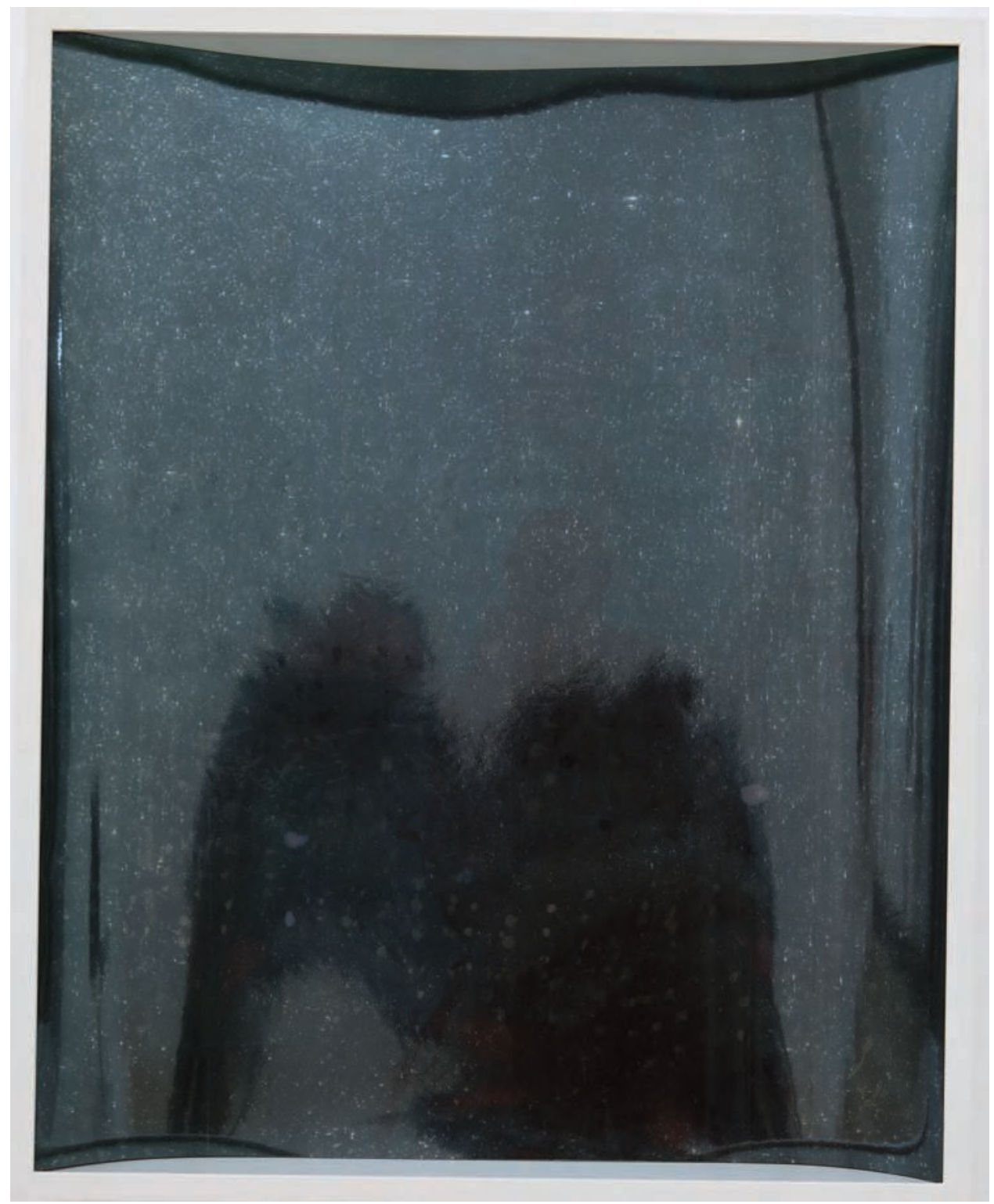

13. Liz Deschenes: Untitled (Bracket), 2015 (tirage gélatino-argentique, encre argent).

Adepte de la photographie sans appareil, l'artiste produit un photogramme en exposant une feuille de papier photosensible à la lumière ambiante avant de la rincer avec un toner argenté - un processus dépendant de la température et de l'humidité, qui ne cesse de se développer par oxydation. 
14. Jean-Luc Moulène : Noud soufflé, 2012 (verre, métal). Au CIRVA, Jean-Luc Moulène a produit une série de nœuds en verre, qui ont obligé les souffleurs à redoubler d'ingéniosité ; ainsi seul le filet noir atteste des circonvolutions auxquelles ils se sont livrés pour entrecroiser leurs boudins en fusion.

15. Outils rustiques Pas de vis (écrou) et vis centrale de pressoir (bois, acier) ; tas étampe en fonte d'acier (date inconnue). Ces étranges outils de bois et d'acier n'ont peut-être pas tout à fait perdu leur valeur d'usage ; mais leur forme est si particulière qu'ils fascinent les sculpteurs contemporains.
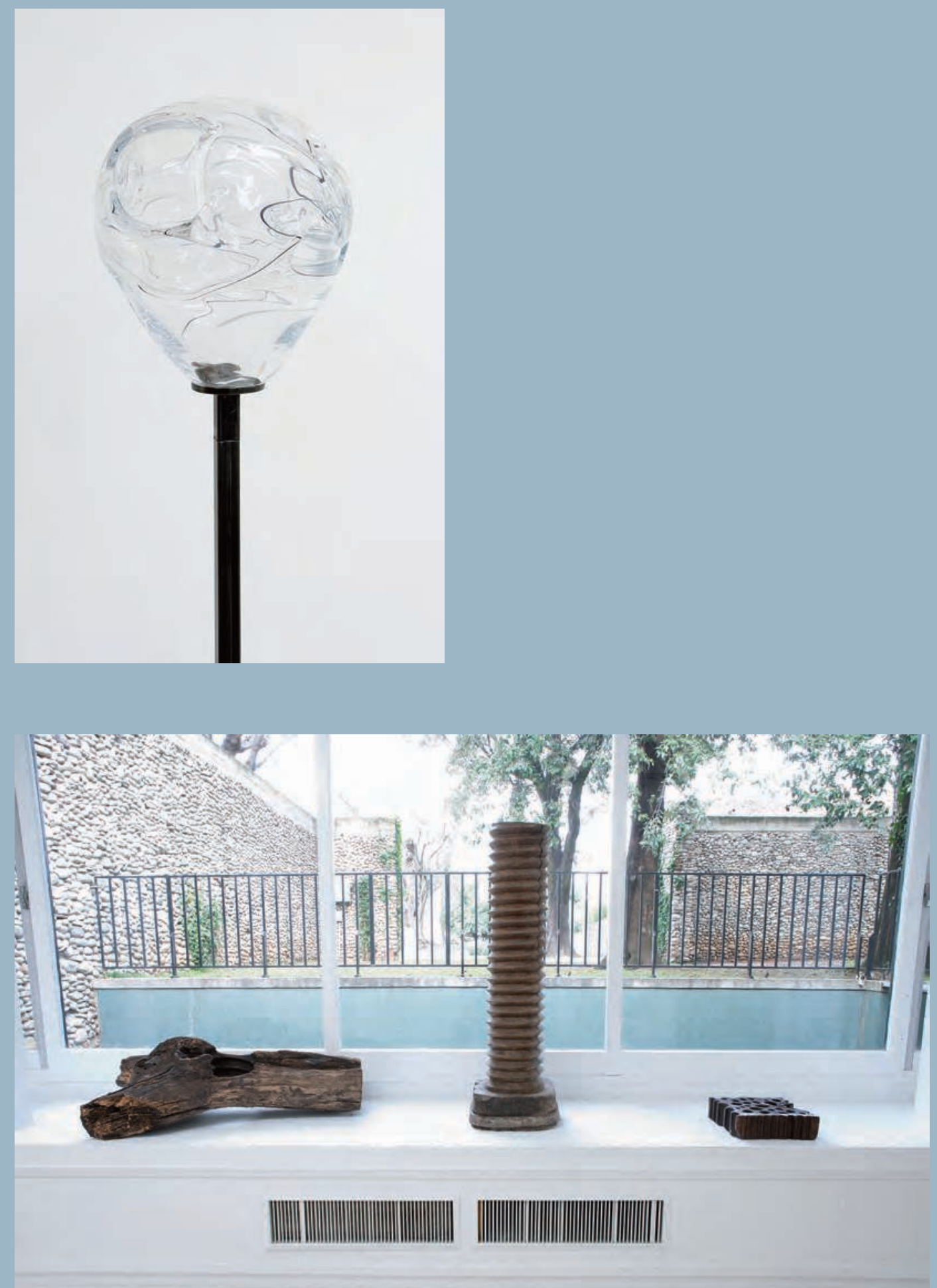


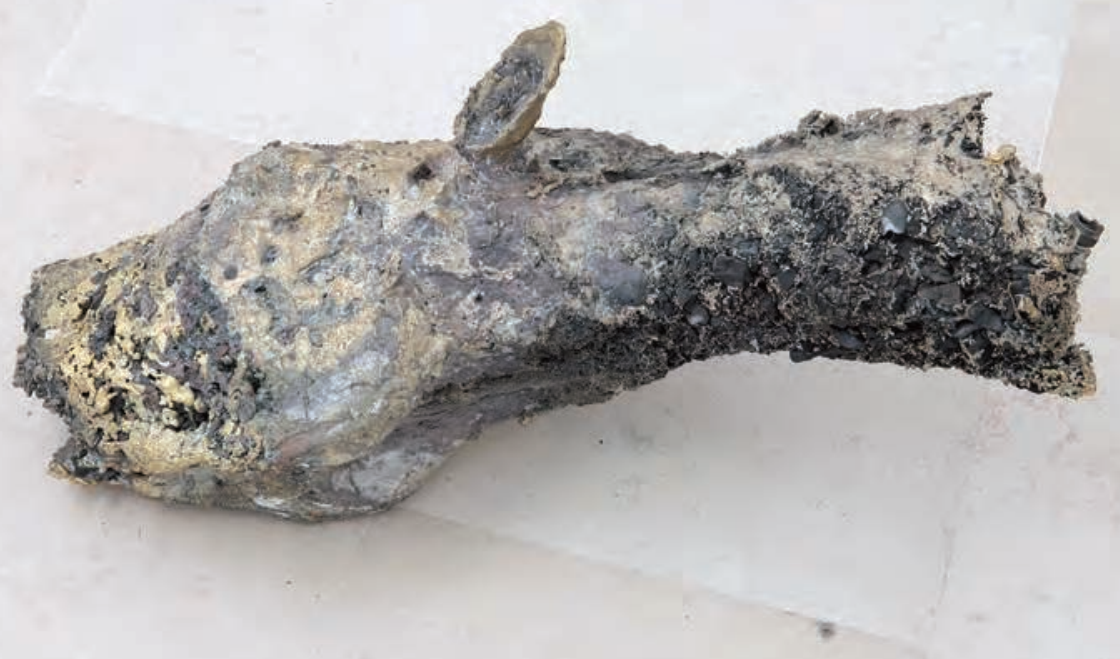

6. Jean-Marie Perdrix : Cheval, bronze à la chair perdue - 3, 2013 (fonte d'un alliage cuivré, charbon et cendres). Perdrix travaille avec des bronziers du Burkina Faso. II a inventé un procédé de fonte du bronze en récupérant des carcasses d'animaux abandonnés : il coule le bronze sur la carcasse même, qui fond comme de la cire, laissant place à sa « dépouille » métallique.
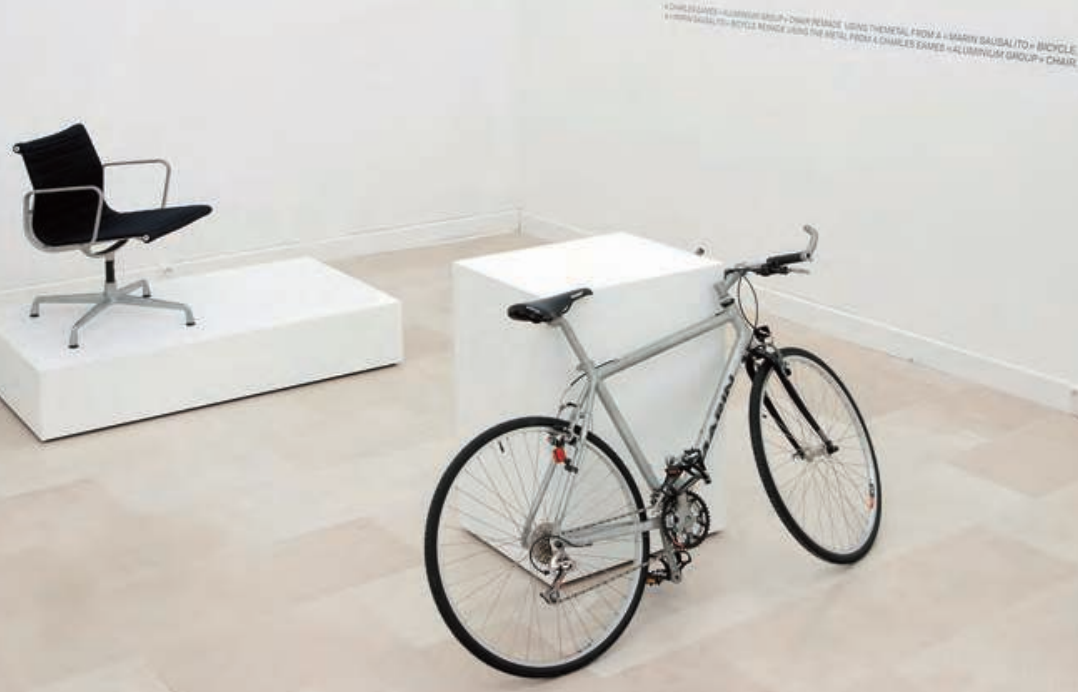

17. Simon Starling Work, Made-Ready, Kunsthalle Bern, 1996 (installation avec bicyclette, chaise en aluminium, bois et vinyle). L'aluminium du fauteuil Eames est utilisé pour faire le vélo Marin Sausolito, et réciproquement. Par cette déconstruction manuelle, Starling réhumanise deux objets produits industriellement. 


\section{Projet}

Lart conceptuel, terme forgé par Sol LeWitt en 1967, semble avoir renoué avec une tradition théorique qui remonte à Aristote. Pour le philosophe grec, toute production d'objet ou d'image relève d'un travail d'actualisation d'une forme, pensée dans l'esprit du producteur, dans la matière passive, au mieux résistante. L'art conceptuel prend à la lettre cette théorie et situe dans le moment où l'esprit conçoit la forme l'essentiel du travail artistique : la matérialisation de la forme n'est qu'un problème d'artisanat. Cependant les formes artistiques, même conceptuelles, possèdent également une matérialité : un texte reste écrit sur un support, un projet peut apparaître comme une maquette ou un dessin. Le grand intérêt des propositions conceptuelles présentées ici ne relève pas de leur réduction de l'art à l'idée, mais de l'inventivité dans la manière de présenter des projets de pièces, qui deviennent pièces eux-mêmes.

18. Sol LeWitt : Wall Drawing \# 172: Lines throught the center of the wall toward midpoints of sides and corners, première installation à la Galerie Lisson de Londres, 1973 (crayon noir Caran D'Ache Neo Color II: crayons solubles à l'eau). Si l'intention initiale de l'artiste résidait dans le don de ses protocoles de dessin à quiconque serait capable de les exécuter, leur réalisation dorénavant passe par l'aval de la Fondation Sol LeWitt, qui contrôle et normalise leur production.

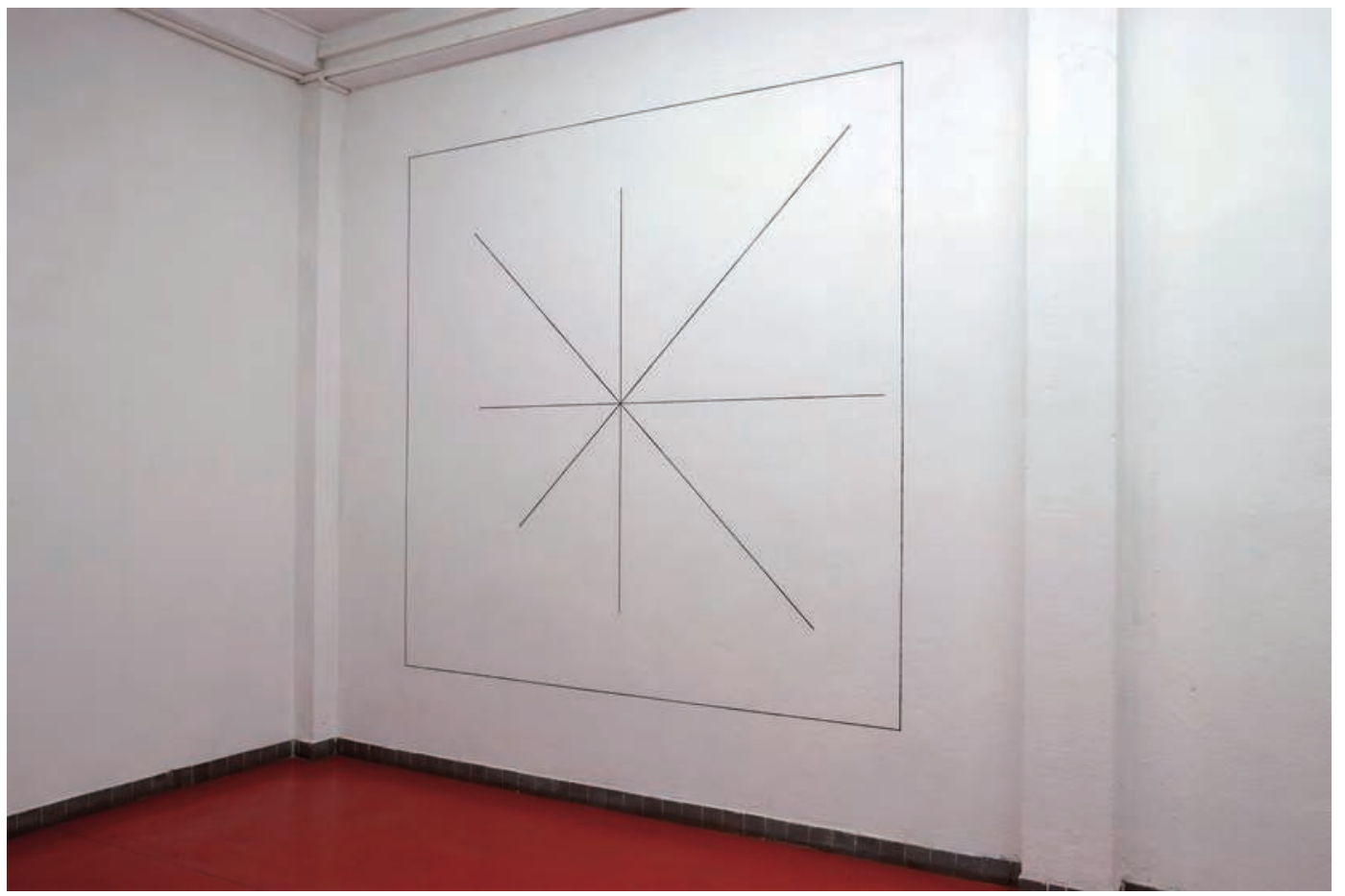




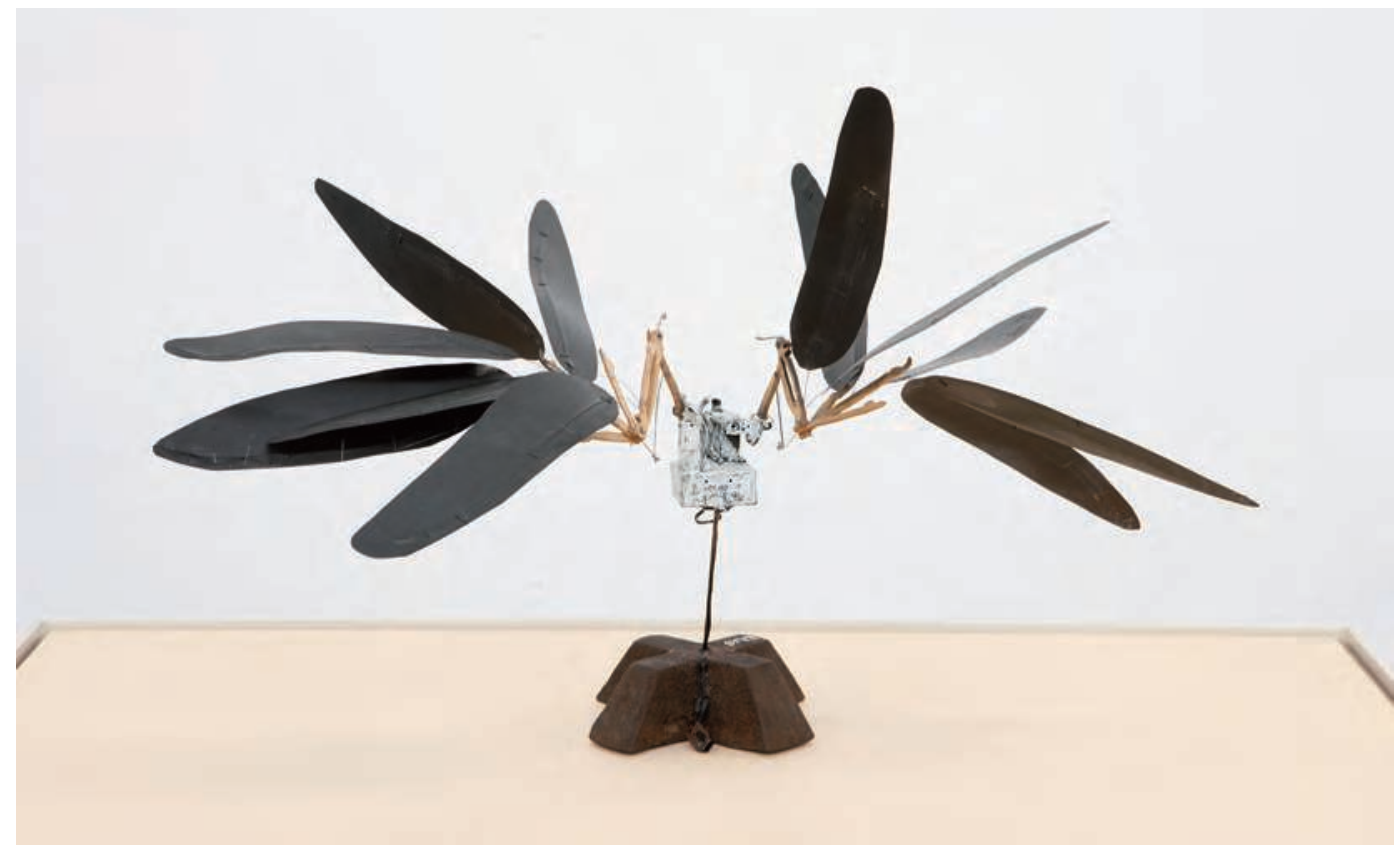

19. Panamarenko: Raaf (Corbeau), 1997 (matériaux divers). Fasciné par le vol des oiseaux, Panamarenko a passé sa vie à produire des prototypes d'objets volants. Ici les ailes noires de la machine lui donnent son nom d'oiseau, même si le souci du détail manifeste l'intérêt de l'artiste pour une poétique du bricolage.

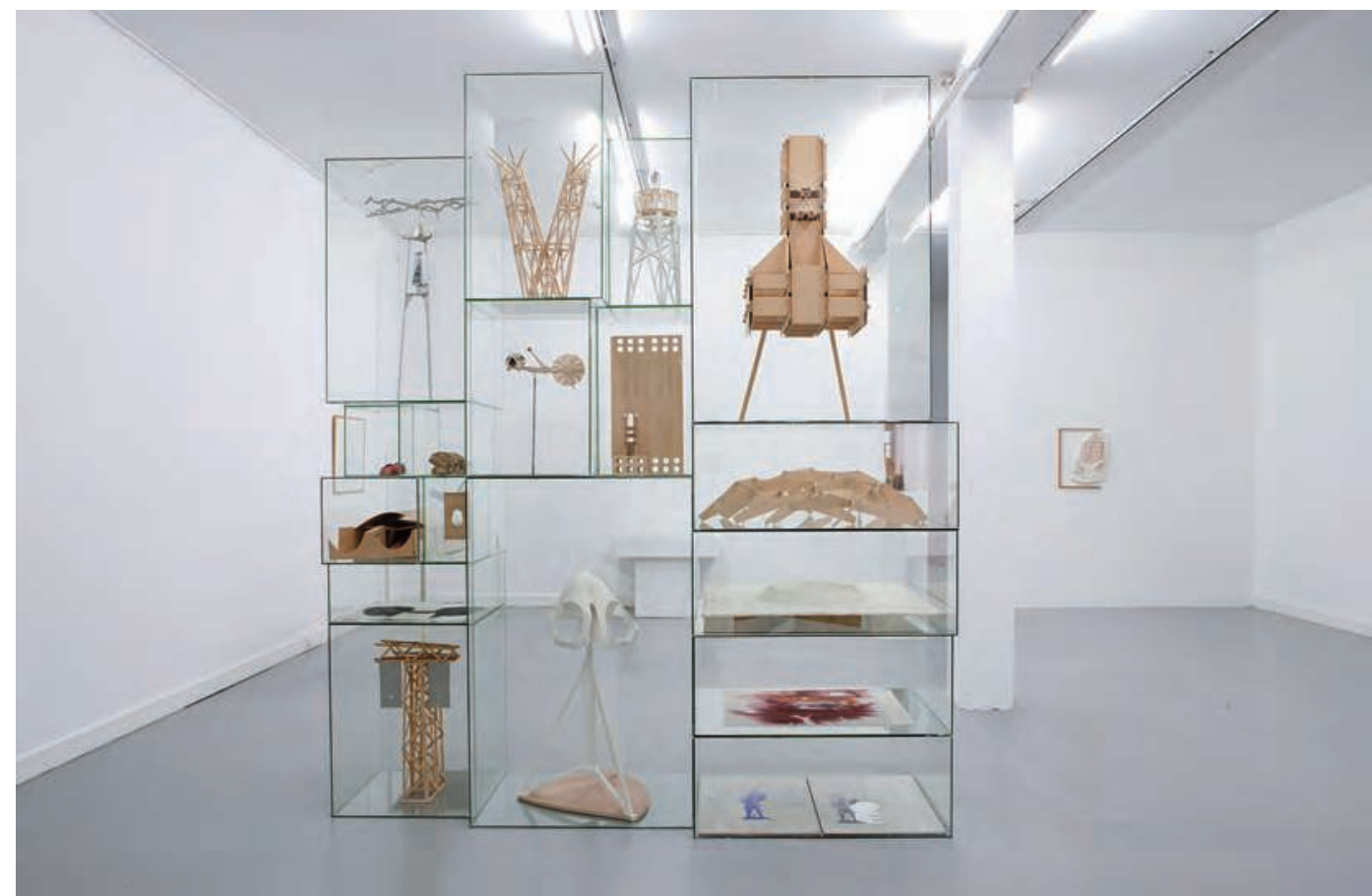

20. Bernhard Rüdiger : Atlas nouveau pour XXe FIN, 1997-2015 (matériaux divers). Rüdiger présente depuis quelques années les maquettes de ses sculptures dans un assemblage qu'il nomme « atlas », en référence à Aby Warburg. II entend ainsi donner forme aux ramifications multiples de sa pensée créatrice. 


\section{Technologie}

Par «technologie », on entend ici une catégorie de machines dont le fonctionnement repose sur un mécanisme complexe. L'automate fait ici figure de modèle antique mais, dans le sens courant, c'est plutôt au monde industriel que renvoie la technologie. Un artiste qui fait usage de la technologie dans sa pratique s'inscrit donc forcément dans une histoire relativement récente de la technique. Encore faut-il distinguer les artistes qui travaillent avec, par exemple, des machines électriques (comme une caméra vidéo ou une perceuse) et ceux qui travaillent sur de telles machines. Chez ces derniers, les machines électriques ne sont pas de simples outils, mais les enjeux de leur recherche artistique : ils en explorent les propriétés, les possibilités qui n'apparaissent pas dans leur usage courant. On pourrait dire qu'ils les élèvent et les observent comme des animaux rares, mais parfois les hybrident pour créer des monstres.

Fabien Giraud \& Raphaël Siboni : La Mesure Louvre, 2011 (vidéo HD, 40 minutes). Le film est un long travelling sur l'accélérateur de particules du Musée du Louvre, qui analyse la composition des sculptures et des tableaux anciens. Les artistes mettent en scène la confrontation de l'œil de la caméra et de celui de l'énorme machine.

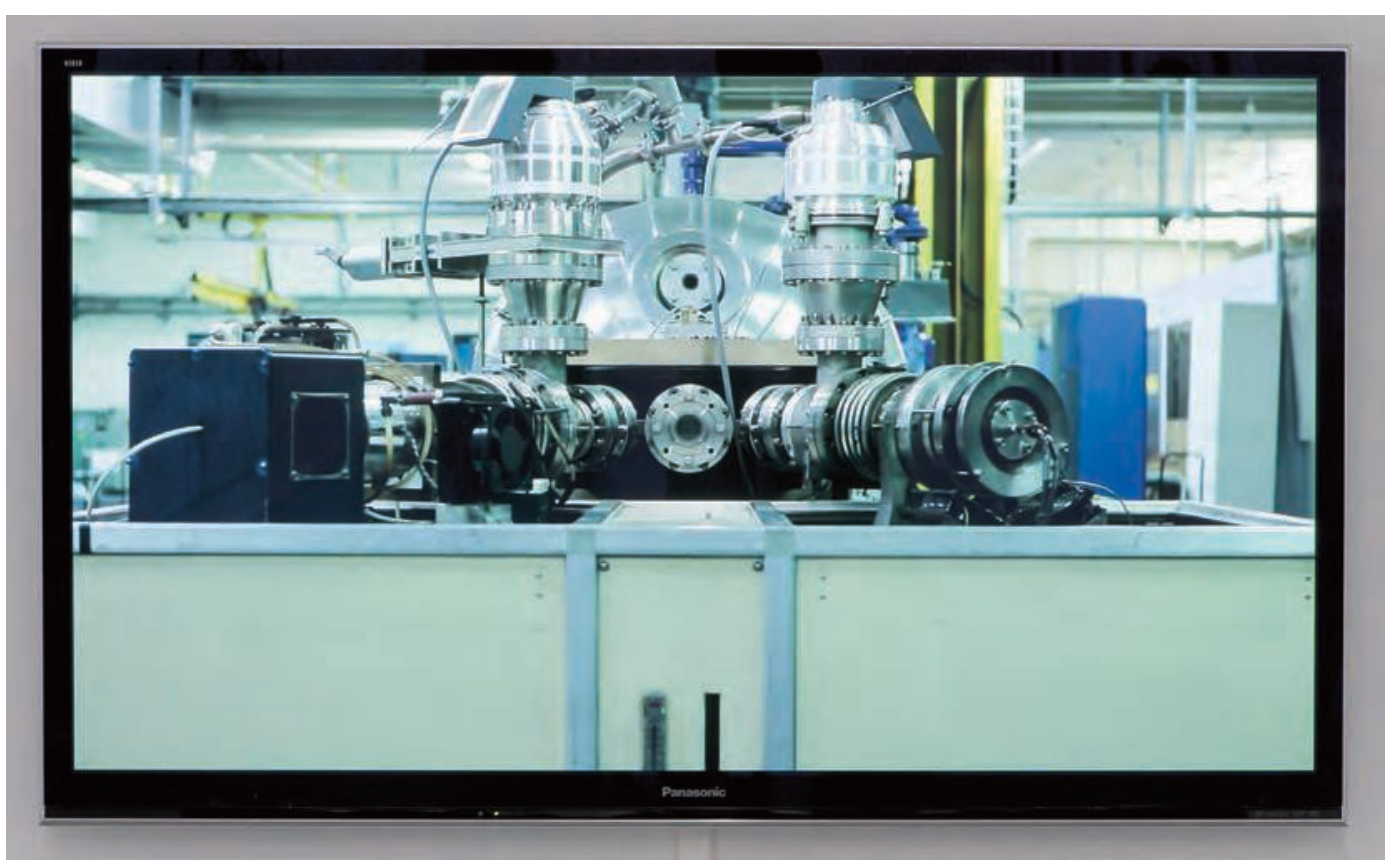




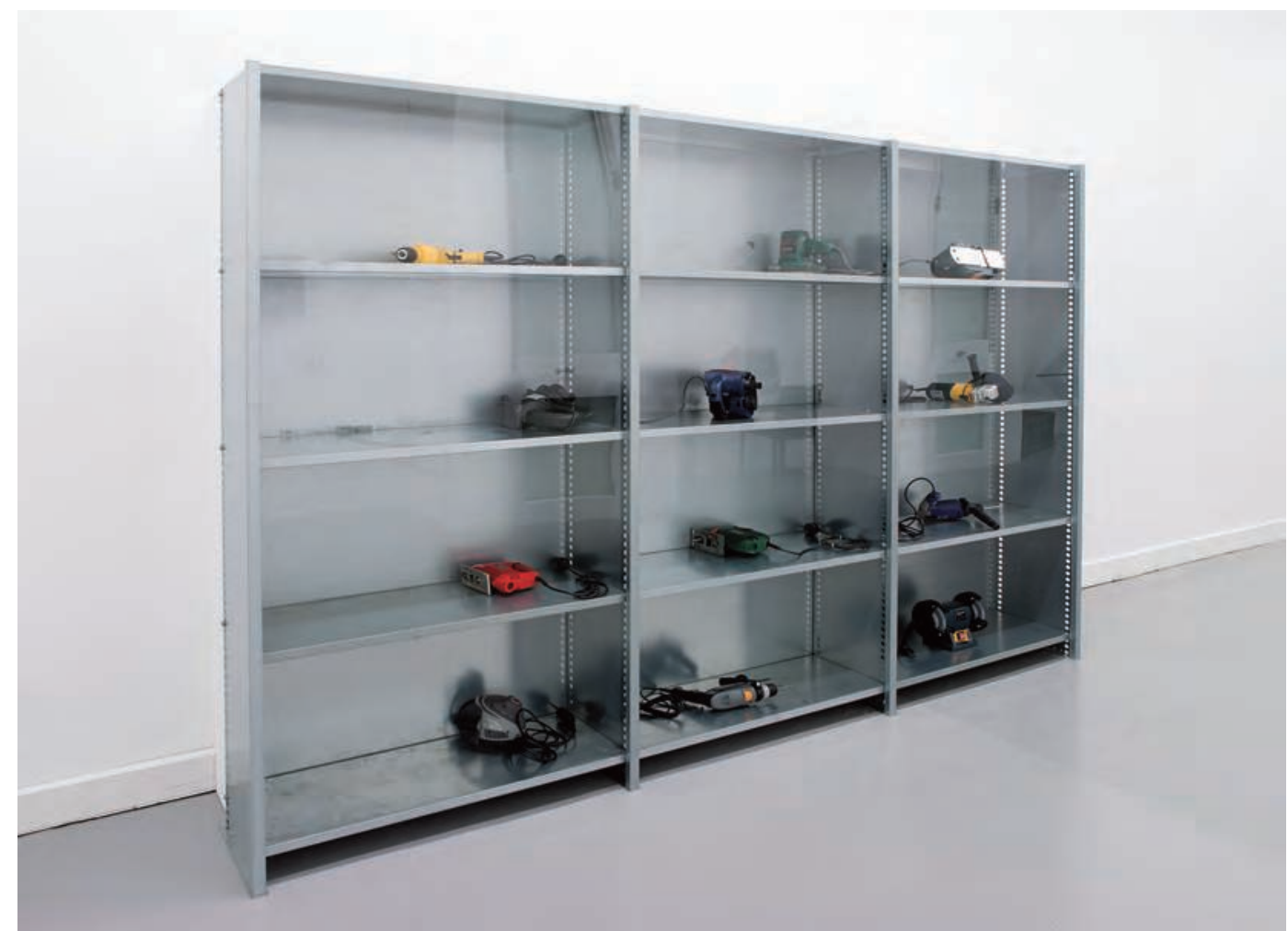

22. Delphine Reist Étagères, 2007 (étagères métalliques en acier galvanisé vitrées en plexiglas, outils électriques, système électronique de régulation). Une collection de perceuses, scies, semi-professionnelles, qu'on peut trouver dans les grandes enseignes de bricolage, s'animent alternativement, telles des animaux en cage : l'outil devient vivant, prend son autonomie et dicte ses choix à l'artiste.

\section{Virtuosité}

Virtus en latin signifie excellence et ce n'est que sous l'effet du christianisme que la vertu a pris la connotation morale qu'on lui confère aujourd'hui. Un artiste vertueux a le sens du bien, un technicien virtuose pousse son savoir-faire à ses limites. Mais souvent on reproche à l'adepte du « hard craft » (l'artisanat virtuose) d'exercer son art dans les ornements de surface et de manquer de profondeur, de sensibilité. Cette opposition - classique - s'effondre dès lors que les démarches de l'artiste et de l'artisan ne se contredisent plus a priori, mais répondent à une recherche commune : le sens du défi, qui peut parfois prendre un tour humoristique. L'ornementation peut alors obéir à une logique intellectuelle autant que formelle. 


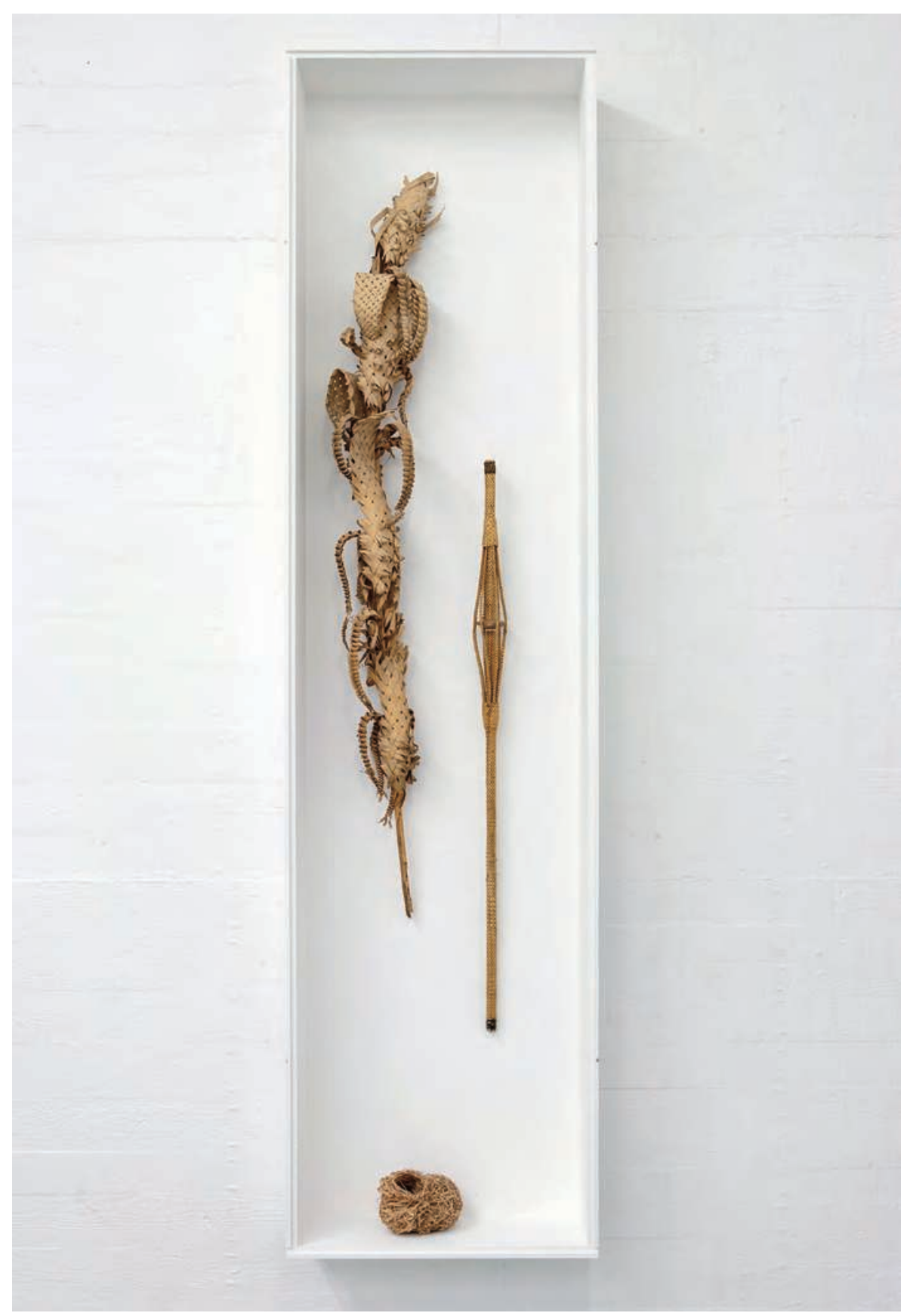

23. Vitrine de la virtuosité : Nid d'oiseau; Quenouille tressée, xIxe siècle (originaire du Piémont);

Épi tressé, date inconnue (fibres végétales). Quoi de commun entre un épi tressé niçois, fabriqué pour les Rameaux, une quenouille traditionnelle des Alpes et un nid d'oiseau tisserin de l'île Rodrigues? Une évidente virtuosité dans le tressage. 


\section{I'auteur}

Thomas Golsenne est professeur d'histoire des arts visuels à la Villa Arson (Nice), docteur en histoire de l'art et ancien pensionnaire de l'Académie de France à Rome. Il a notamment co-publié une nouvelle traduction en français du De Pictura de Leon Battista Alberti (2004), co-dirigé La performance des images (2010), écrit Pascal Pinaud, serial painter (2015) et a publié divers articles sur l'ornementalité à la Renaissance ou dans l'art contemporain, sur l'anthropologie des images, les formes occidentales de l'animisme ou sur la théorie de l'art. Il coordonne avec Patricia Ribault ce numéro 64 de TechniquesÉCulture consacré à l'ethnologie de l'art et du design contemporains.

\section{Iconographie}

Image d'ouverture. S-y-n-d-i-c-a-t (François Havegeer, Sacha Léopold), affiche de l'exposition Bricologie. La souris et le perroquet, 2015, Villa Arson, Nice.

1. Alexandre Ovize et Florentine Larmarche : Potacrayons, 2014 (40 x 40 × 70 cm, Galerie Luis Adelantado, Valencia) et Lithographies, 2014 (édition de 20, production URDLA, Villeurbanne, 56 x $76 \mathrm{~cm}$ ), Galerie Luis Adelantado, Valencia. 2. Barbara Schrobenhauser : Aluminium II (haut : $29 \mathrm{~cm}$, pendentif: 3,3 x 5,6 × 4,8 cm, numéro 2 d'une édition de 3), courtesy de l'artiste @ photographie Thomas Golsenne. 3. Thomas Thwaites: The Toaster Project, 2011.

4. Contrebassine (125 x 41 x 50 cm), Musée Communautaire des Arts et Traditions Populaires, Draguignan.

5. Marcel Duchamp : Porte, 11 rue Larrey, 1927, production Villa Arson

6. Robert Filliou : Briquolage I, 1982 (dimensions variables), Carré d'art - Musée d'art contemporain, Nîmes.

7. Guillaume Gouérou :Metatron Projec - MW6400, $2013-$ 2014 (dimensions variables), courtesy de l'artiste.

8. Sofia Hultén : Altered Fates, 2013, Galerie Daniel Marzona/Raebervon Stenglin, Galerie Nordenhake, courtesy de l'artiste.

9. Arnaud Vasseux : Creux, 2011-2013 (dimensions max/ min d'un creux : 2 à 3,5 x 6,5 cm et de 9 à 14,5 x 6,5 cm), Collection Centre International de Recherche sur le Verre et les Arts Plastiques CIRVA, Marseille.

10. Vitrine du geste : statue de saint Marc de Notre-Dame de Brusc $(61 \times 31 \times 18 \mathrm{~cm})$ et silex taillé $(20 \times 9 \times 5 \mathrm{~cm})$, Collection Musée d'Art et d'Histoire de Provence, Grasse ; massette en métal, Musée des métiers traditionnels de Tourrette-Levens.

11. Dominique Blais : Entropê, 2014-2015 (partie verre : H 23,50 cm x $\varnothing 14,50 \mathrm{~cm}$ - socle : 110 x 55 x 55 cm). Réalisation: CIRVA, Marseille (verre)/Atelier Marc Descarrega, assisté de Marine Anglard (ébénisterie), Galerie Xippas, Paris, courtesy de l'artiste.
12. Xavier Antin : Sans titre (An Epoch of Rest), 2014 $(150 \times 150 \times 50 \mathrm{~cm})$ et Sans titre (An Epoch of Rest), 2015 $(180$ x 80 x 60 cm), Galerie Crèvecœur, Paris.

13. Liz Deschenes: Untitled (Bracket), 2015 (98.7 x $79.7 \times 16.5$ $\mathrm{cm}$, encadré), Galerie Campoli Presti, Londres/Paris.

14. Jean-Luc Moulène, Noud soufflé, 2012 (145 x 25 x 25 $\mathrm{cm})$, réalisation : CIRVA, Marseille (c) CIRVA.

15. Outils rustiques : pas de vis $(125 \times 70 \times 43 \mathrm{~cm})$ et vis centrale de pressoir $(138 \times 45 \times 45 \mathrm{~cm})$, Musée Communautaire des Arts et Traditions Populaires, Draguignan ; tas étampe en fonte d'acier, Musée des métiers traditionnels de Tourrette-Levens.

16. Jean-Marie Perdrix : Cheval, bronze à la chair perdue -3, 2013 ( 28 x 112 x 25 cm), Collection de l'artiste.

17. Simon Starling : Work, Made-Ready, Kunsthalle Bern, 1996 (dimensions variables), Collection du Fonds Régional d'Art Contemporain Languedoc-Roussillon.

18. Sol LeWitt: Wall Drawing \# 172: Lines throught the center of the wall toward midpoints of sides and corners, 1973, The LeWitt Collection, Chester, CT.

19. Panamarenko : Raaf (Corbeau), 1997 (30 × 57 × 36 cm), Collection Antoine de Galbert, Paris.

20. Bernhard Rüdiger : Atlas nouveau pour XXe FIN, 1997$2015(300 \times 250 \times 70 \mathrm{~cm})$, Collection de l'artiste.

21. Fabien Giraud \& Raphaël Siboni : La Mesure Louvre, 2011, Galerie Loevenbruck, Paris.

22. Delphine Reist : Étagères, 2007, Collection Institut d'Art Contemporain, Rhône-Alpes.

23. Vitrine de la virtuosité : Nid d'oiseau, Collection particulière ; Quenouille tressée, XIx ${ }^{e}$ siècle $(6,4 \times 106,3$ $\mathrm{cm})$, Collection du Musée Museum Départemental des Hautes-Alpes, Gap ; Épi tressé, date inconnue (142 x 15 x 15 cm), Musée Communautaire des Arts et Traditions Populaires, Draguignan.

Crédits photographiques pour l'ensemble des images : (C) Jean Brasille / Villa Arson sauf mention contraire. 


\section{Références}

Detienne, M. \&Vernant, J.-P. 1974 Les Ruses de l'intelligence, la Mètis des Grecs, Paris : Flammarion.

Focillon, H. 2013 [1934] Vie des formes suivi de Éloge de la main, Paris : PUF.

Frontisi-Ducroux, F. 2000 [1975] Dédale : mythologie de l'artisan en Grèce ancienne, Paris : La Découverte.

Gell, A. 1992 « The Technology of Enchantment and the Enchantment of Technology » in Coote, J. \& Shelton, A. (eds.), Anthropology, Art and Aesthetic, Oxford : Clarendon Press : 40-63.

Lévi-Strauss, C. 1962 La Pensée sauvage, Paris : Plon. Simondon, G. 2014 [1982] « Réflexions sur la techno-esthétique » in Sur la technique, Paris : PUF : 379-396.

\section{Pour citer cet article}

Golsenne, T. 2015 « Bricologie. La souris et le perroquet. Retour sur une exposition », Techniques\& Culture nº 64 « Essais de Bricologie. Ethnologie de l'art et du design contemporains », p. 128-151. 